\title{
CRISPR-based technologies for cell biology
}

\author{
Advances in CRISPR-based systems have greatly expanded the molecular toolbox for biologists. In this issue, we \\ present the first of a Series of commissioned Review articles that highlight the progress made using CRISPR-Cas 9 \\ technology and its relevance for cell biological research.
}

( riginally identified as part of the adaptive immune system of prokaryotes, researchers were quick to recognise the potential of clustered regularly interspaced short palindromic repeats (CRISPR) and the associated endonuclease Cas9 for efficient genome editing. The CRISPR-Cas9 system is RNA guided and cleaves the DNA double strand, providing a powerful method to induce deletions, insertions and specific sequence changes at defined target sites. The comparable ease of manipulating the guide RNA represents an advantage over other customizable nucleases, such as zinc finger and transcription activator-like effector nucleases. CRISPR-Cas9 efficiently edits the genome in a wide variety of cell types and whole organisms, and its preciseness and ease of use have spurred its widespread adoption in biological research. Modified versions of the technology have added extra functions to the gene-editing tool, such as the regulation of endogenous gene expression, the modulation of the epigenetic status and the labelling of genomic loci.

Ever since the CRISPR-Cas9 system was first adapted for use in targeted genome editing in 2012, the scientific community has made remarkable progress in improving efficiency and accuracy of editing, expanding the list of applications and targetable species. Importantly, the platform has been used to explore fundamental cell biological questions, and we are delighted to highlight some of these accomplishments in our Series on CRISPR technology, launched in this issue of Nature Cell Biology. Alongside the first two commissioned Perspective and Review articles in this Series, hosted on a dedicated page of our website (https://www.nature. com/collections/igbbbjccfa), readers can also access an online collection featuring related research published in Nature Cell Biology. The Series will be updated as new primary and commissioned content is published.

In a Perspective article in this issue, Lea and Niakan provide a comprehensive overview of the use of CRISPR-Cas9 in human germline genome editing to date. Efforts to improve our understanding of early human development are particularly benefiting from this technology, as it allows the instant readout of functional consequences of targeted gene disruption. Another aim is to preclinically assess the feasibility of CRISPR-Cas9 systems to correct pathological mutations in human embryos. As highlighted by the authors, new insights have been gained in both areas, but many challenges remain, such as the possibility of off-target effects or large DNA deletions and rearrangements after editing. CRISPR-Cas9mediated mutagenesis may lead to abnormal development or promote embryo arrest if not repaired correctly. The consequences of using different DNA repair pathways for genome editing are also further described in a Review in this Series, discussed below.

In the second part of this Perspective article, Lea and Niakan consider potential clinical and ethical implications of this technology. In particular, the announcement of CRISPR-Cas9-edited babies during the Second International Summit on Human Genome Editing in Hong Kong in 2019 has re-ignited the debate about a moratorium on human germline genome editing. The authors set out a list of key prerequisites that will have to be met to ensure the safe and ethical use of this technology for clinical human embryo genome editing in the future.

The Review article by Yeh, Richardson and Corn focuses on the DNA repair pathways that resolve DNA damage during genome editing and how they can entail different editing outcomes. Generally, doublestranded breaks (DSBs) are repaired through re-ligation of the strand ends, for instance in non-homologous end joining (NHEJ), which can induce insertions or deletions, or through homology-directed DNA repair (HDR), a method that requires a donor DNA template to guide the repair. Both pathways have been a matter of extensive debate in genome editing, as they ultimately achieve different editing results. Most work to date focuses on HDR to introduce designed edits into human cells, which is generally seen as less errorprone than NHEJ but difficult to achieve in human cells.

The authors describe repair pathways, their mechanism of action, advantages and limitations and give an excellent overview of available molecules to control gene-editing results by manipulating DNA repair. Different cell cycle phases can activate separate repair pathways, which means that timed delivery of CRISPR-Cas9 during phases that favour HDR can increase the efficiency of editing. Even so, HDR comes with its own set of technical limitations that limit repair efficiency and increase the risk of unintended mutations, as also discussed by Lea and Niakan in the context of human germline genome editing. Base editing or the most recently reported primed editing (Nature https://doi.org/10.1038/s41586-0191711-4; 2019) could circumvent the need for DSBs, HDR and donor templates. The latter method uses a Cas9 nickase that is fused to an engineered reverse transcriptase and a prime editing guide RNA, but so far has only been tested in human cell lines.

Upcoming pieces in the Series will include a Perspective article on RNAtargeting CRISPR-Cas tools by Gene Yeo and colleagues, discussing how this technology translates into applications for RNA biology. Over the subsequent months, we hope to present additional Review articles, for instance, covering modified CRISPR-based platforms that enable 3D manipulation of the genome structure and its nuclear position.

Innovative CRISPR-based technologies are being developed at a remarkable pace, and the diverse nature of engineering solutions offers steadily increasing functionality for the toolbox. Many of the resourceful applications made possible by additional CRISPR-Cas9 modifications are directly relevant to and dependent on the study of classical cell biological areas, such as DNA repair, cell cycle, RNA biology, nuclear organisation, and provide fascinating new insights into human disease and developmental biology. In this spirit, we look forward to the discoveries and methodological advances that still lie ahead in this stimulating field of research. We thank our authors and referees for their contributions, and hope that this Series will provide both valuable information and inspiration for our readers.

Published online: 2 December 2019 https://doi.org/10.1038/s41556-019-0434-y 